\title{
A retrospective analysis of ureteropelvic junction obstructions in patients with horseshoe kidney
}

\author{
Mohamed Ibrahim Ahmed Elmaadawy, ${ }^{1,2}$, Sang Woon Kim ${ }^{1}$, Sung Ku Kang ${ }^{3}$, Sang Won Han ${ }^{1}$, \\ Yong Seung Lee ${ }^{1}$
}

${ }^{1}$ Department of Urology and Urological Science Institute, Yonsei University College of Medicine, Seoul, Republic of Korea; ${ }^{2}$ Department of Urology, Faculty of Medicine, Tanta University, Tanta, Egypt; ${ }^{3}$ Department of Urology, National Health Insurance Service Ilsan Hospital, Goyang, Republic of Korea

Contributions: (I) Conception and design: YS Lee; (II) Administrative support: YS Lee; (III) Provision of study materials or patients: SW Kim, SW Han, YS Lee; (IV) Collection and assembly of data: MIA Elmaadawy, SK Kang; (V) Data analysis and interpretation: MIA Elmaadawy, SK Kang, YS Lee; (VI) Manuscript writing: All authors; (VII) Final approval of manuscript: All authors.

Correspondence to: Yong Seung Lee. Department of Urology, Yonsei University College of Medicine, Seoul, Republic of Korea.

Email: asforthelord@yuhs.ac.

\begin{abstract}
Background: Ureteropelvic junction obstruction (UPJO) is often encountered in patients with a horseshoe kidney (HSK) and may require surgical intervention. This study retrospectively investigated obstruction causes in HSK patients with UPJOs to determine the most suitable surgical method.

Methods: Twenty HSK patients with UPJO who underwent pyeloplasty between July 2000 and June 2020 and were followed-up for more than six months in our institution were included in the study. The clinical characteristics, obstruction causes, and surgical outcomes were analyzed.

Results: The median age at the time of the operation was 4.1 years [interquartile range (IQR): 1.8-10.6]. Hydronephrosis (HN) was found prenatally in 5 patients $(25.0 \%)$. Pyeloplasty was performed by open, laparoscopic, and robotic techniques in 6, 10, and 4 patients, respectively. Sixteen patients $(80.0 \%)$ had high ureteral insertion. Twelve patients $(60.0 \%)$ had crossing vessels, and eight had a high ureteral insertion and crossing vessels. The median follow-up duration was 4.0 years (IQR: 1.8-8.9); no patient required additional surgery. The median differential renal function was 38.0\% (IQR: 16.9-43.0\%) preoperatively and 38.0\% (IQR: $13.3-48.2 \%$ ) postoperatively.

Conclusions: UPJOs in HSKs were primarily caused by a high ureteral insertion and crossing vessels. Dismembered pyeloplasty was successfully performed in all surgical modalities such as the open, laparoscopic, and robotic approaches. Attention must be given to patients with HSKs, even in those without $\mathrm{HN}$, to avoid UPJO development.
\end{abstract}

Keywords: Fused kidney; hydronephrosis; child; kidney pelvis; laparoscopy

Submitted Jun 03, 2021. Accepted for publication Oct 15, 2021.

doi: $10.21037 /$ tau-21-471

View this article at: https://dx.doi.org/10.21037/tau-21-471

\section{Introduction}

The horseshoe kidney (HSK) is the most documented renal fusion and malrotation anomaly, occurring in approximately 1 in every 500 individuals (1). It was first described in early autopsy studies by De Carbi in 1522 and discussed in greater detail by Morgagni in 1720 (2). HSKs may be associated with other urinary tract abnormalities, including ureteropelvic junction obstruction (UPJO), vesicoureteral reflux, and a duplex collecting system (3). Most HSK cases are asymptomatic and surgical intervention is not required unless there is complicated UPJO (4). Although various structural anomalies cause UPJOs in HSKs, a comprehensive analysis has never been performed. As a 
result, various surgical methods have been presented in case reports. This study retrospectively investigated obstruction causes in HSK patients with UPJOs to suggest the most suitable surgical method.

We present the following article in accordance with the STROBE reporting checklist (available at https://dx.doi. org/10.21037/tau-21-471).

\section{Methods}

This study was conducted in accordance with the Declaration of Helsinki (as revised in 2013). The study was approved by institutional review board of Severance Hospital (4-2020-1078) and individual consent for this retrospective analysis was waived. Patients with HSK who underwent pyeloplasty between July 2000 and June 2020 and were followed-up for more than six months in our institution were included.

\section{Patients}

Initially, a conservative treatment plan with regular ultrasonography and diuretic renography was implemented when HSK patients were diagnosed with UPJO. Pyeloplasty was considered for treatment when there was more than a $5 \%$ decrease in differential renal function (DRF), hydronephrosis worsened (HN), urinary tract infections (UTIs) recurred, or flank pain developed. The clinical characteristics, obstruction cause, and surgical outcomes were analyzed.

\section{Surgical techniques}

Pyeloplasty was performed with open, laparoscopic, and robot-assisted laparoscopic modalities. Under general anesthesia, retrograde ureteropyelography was performed to check the structure of the ureter and ureteropelvic junction (UPJ). Next, the patient's body position was changed to the semi-lateral position with the ipsilateral side facing up.

For open pyeloplasty $(\mathrm{OP})$, a $1.5-3.0-\mathrm{cm}$ retroperitoneal anterior subcostal flank incision was made. After reaching the retroperitoneal space, the renal pelvis was identified and aspirated to help identify the UPJ and proximal ureter.

Laparoscopic pyeloplasty (LP) and robot-assisted laparoscopic pyeloplasty (RALP) were performed with the transperitoneal approach. In LP cases, one 5-mm camera port was made at the umbilicus, followed by two $3-\mathrm{mm}$ ports for instruments. RALP was performed using the Da
Vinci SI system ${ }^{\circledR}$ or Da Vinci $\mathrm{SP}^{\circledR}$ system (Intuitive Surgical, Sunnyvale, CA, USA). For the SI system, one $8.5-\mathrm{mm}$ camera port was introduced into the peritoneal cavity, followed by two 5 -mm robotic ports and one $5-\mathrm{mm}$ assistant port. For the SP system, the Gel POINT advanced access platform (Applied Medical, Rancho Santa Margarita, CA, USA) was placed through a $3-\mathrm{cm}$ umbilical incision, and a $25-\mathrm{mm}$ port for an articulating camera and two robotic instruments and an additional $12-\mathrm{mm}$ port were introduced through the Gel Seal membrane. Full SP system details are provided in our previous report (5).

All pyeloplasties were completed in a dismembered manner using 7-0 to 6-0 vicryl sutures depending on the diameter of the ureter in an interrupted manner. When high ureteral insertions (HUIs) were encountered, the previous UPJ was ligated or cut with the redundant renal pelvis, and a neo-UPJ was made at the most dependent position of the renal pelvis (Figure 1). When HUIs and crossing vessels (CVs) co-existed, a neo-UPJ was made at the dependent position of the renal pelvis, avoiding the $\mathrm{CV}$ effects. If there were CVs without HUIs, ureter and CV repositioning was performed. In OP, a small Penrose drain was left in the retroperitoneal space with no ureteral stent; in LP and RALP, a double J ureteral stent was inserted anterograde.

\section{Postoperative follow-up}

The urethral catheter was removed on postoperative day 1 . For OP, the Penrose drain remained for at least 3 days until it became dry. For LP and RALP, the ureteric stent was removed after 3-4 weeks. After 4-6 weeks, ultrasonography was performed to evaluate the $\mathrm{HN}$ degree and parenchymal thickness and was repeated after 3-6 months. After 3-12 months, a technetium-99m-mercaptoacetyl triglycine (MAG-3) diuretic renogram was performed to evaluate washout and DRF and was repeated if necessary. Urinalysis and blood pressure checks were performed annually to monitor for proteinuria or hypertension development, and ultrasonography was repeated every 2-3 years. Successful pyeloplasty was defined as an absence of symptoms or complications related to ureter obstruction and $\mathrm{HN}$ improvement.

\section{Statistical analysis}

This is an analysis of rare situations, and the prevalence of each variables was revealed. No statistical method for comparison is used. Continuous variables were expressed 

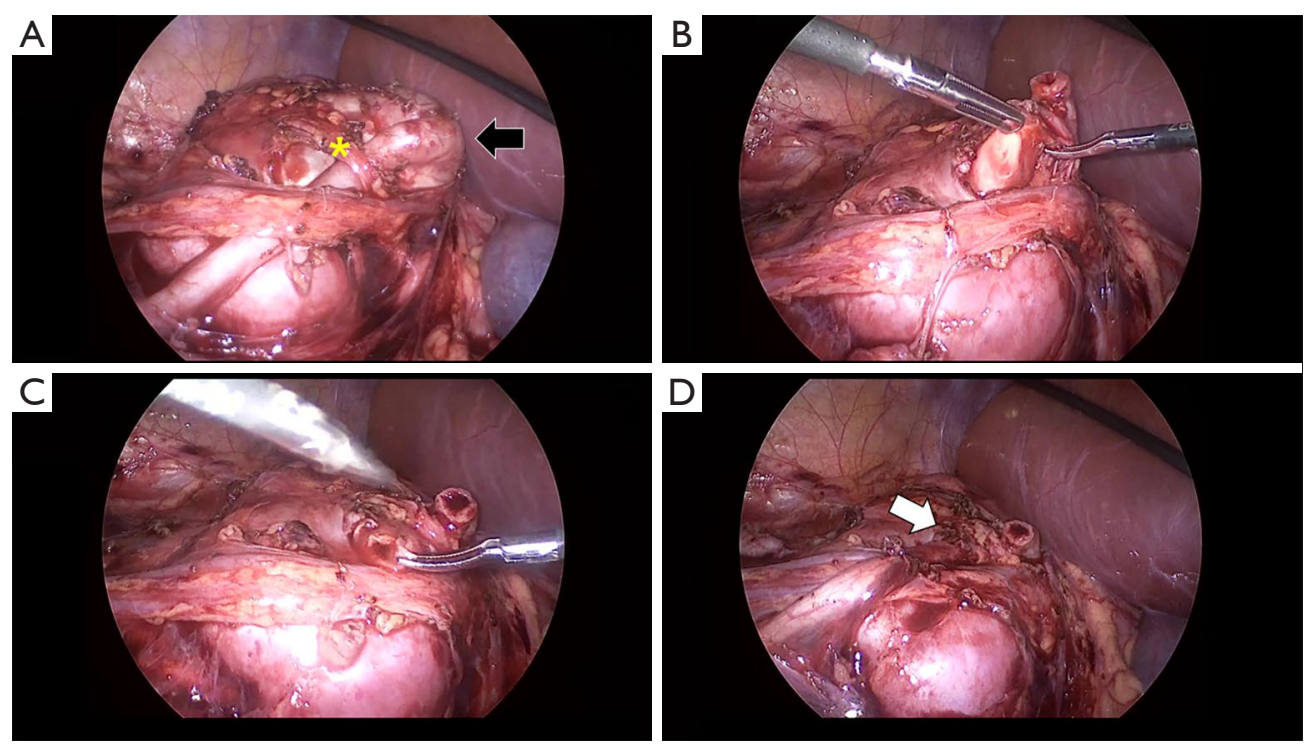

Figure 1 Laparoscopic pyeloplasty images. (A) A crossing vessel (asterisk) with high ureteral insertion (black arrow), (B) ligation of the previous UPJ, (C) making a neo-UPJ, and (D) the neo-UPJ at the most dependent position of the renal pelvis (white arrow).

using median values and interquartile ranges (IQRs).

\section{Results}

One thousand and fifteen patients underwent pyeloplasty during the study period, and $20(2.0 \%)$ had a HSK. The median patient age at the time of the operation was 4.1 years (IQR: 1.8-10.6) (Table 1). HN was diagnosed prenatally in 5 patients $(25.0 \%)$ and after birth in 15 patients $(75.0 \%)$. HSK was diagnosed simultaneously with HN or UPJO in 16 patients $(80.0 \%)$, and HN and HSK were diagnosed during follow-up in 3 patients (15.0\%). HSK without HN was detected prenatally in 1 patient $(5.0 \%)$, but as there was no HN or symptoms, there was no follow-up until flank pain developed at 10.1 years old and ultrasonography revealed HSK and severe HN. Before the operation, renal function deterioration, flank pain development, and recurrent UTIs were observed in $13(65.0 \%), 10$ $(50.0 \%)$, and 6 patients $(30.0 \%)$, respectively. The median preoperative DRF was $38.0 \%$ (IQR: 16.9-43.0).

\section{High ureteral insertion and crossing vessel}

OP was performed in 6 patients $(30.0 \%)$, LP in 10 patients $(50.0 \%)$, and RALP in 4 patients $(20.0 \%)$; RALP was performed with a single port using the Da Vinci $\mathrm{SP}^{\circledR}$ system in 1 patient.
Sixteen patients $(80.0 \%)$ had a HUI, 12 patients $(60.0 \%)$ had CVs, and 8 patients had a HUI and CV (Figure 2). There were no intraoperative complications or surgical conversions (e.g., laparoscopic to open surgery). Fourteen patients who underwent LP or RALP had the ureteral stent removed after 3-4 weeks. Retrograde ureteropyelography was performed immediately after the removal to check for anastomosis leakage and postoperative UPJ features. Twelve of these patients had a preoperative HUI, and postoperative retrograde ureteropyelography showed a UPJ location change to the dependent position of the renal pelvis (Figure 2). Renal isthmus was not divided or separated in all cases as it was found not to be a cause of UPJO. Concomitant renal stone removal was performed in two of our patients during pyeloplasty. In one 9 months old male patient, two $4 \mathrm{~mm}$ renal stones were observed in preoperative ultrasound and were removed during OP. When the pelvis is opened, they were found and removed. In the outer 14 years old male patient, one $4 \mathrm{~mm}$ renal stone was observed in preoperative computerized tomography scan. When the pelvis was opened during RALP, the stone was not found and flexible ureteroscope was inserted through $5 \mathrm{~mm}$ assistant port. The renal stone as then found at the renal pelvis and removed using stone basket.

\section{Postoperative results}

The median number of follow-up years was 4.0 (IQR: 1.8- 
Table 1 Patient characteristics $(\mathrm{N}=20)$

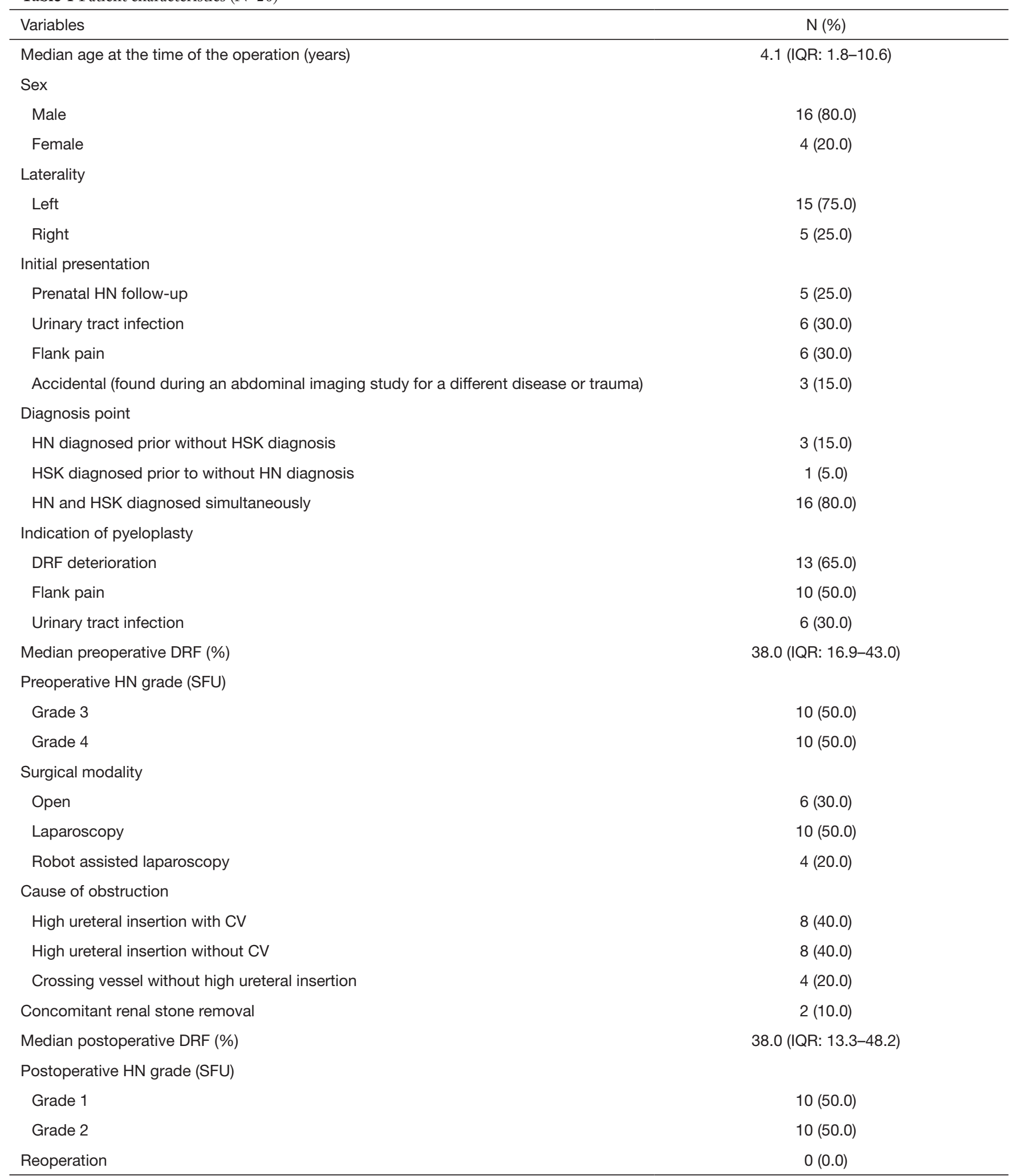

IQR, interquartile range; HN, hydronephrosis; HSK, horseshoe kidney; DRF, differential renal function; SFU, society of fetal urology; CV, crossing vessel. 

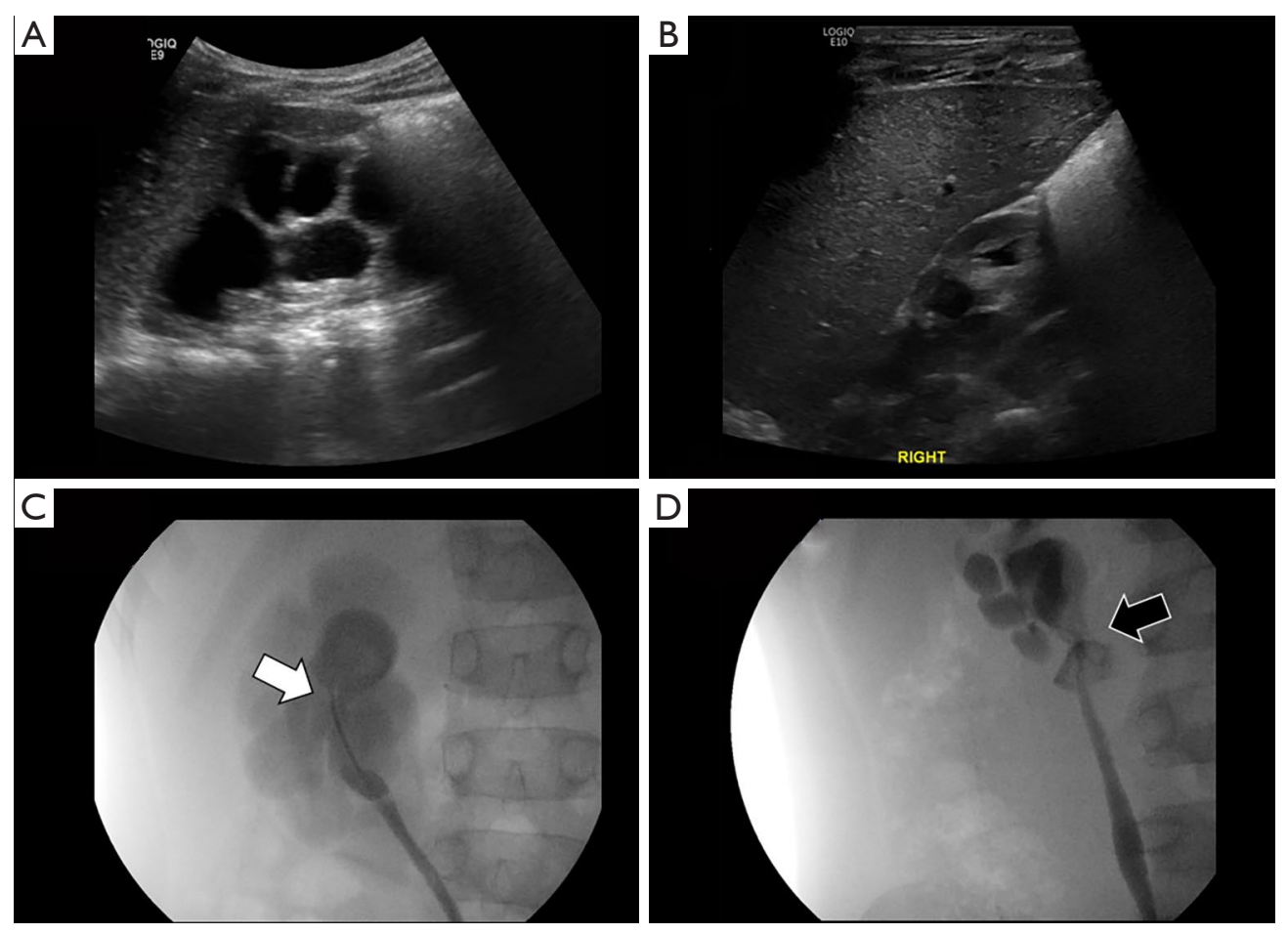

Figure 2 Retrograde ureteropyelography images. (A,B) Postoperative improvement of hydronephrosis, (C) preoperative high ureteral insertion (white arrow), and (D) postoperative imaging after the ureteral stent removal showing that the neo-UPJ was in the dependent position (black arrow).

8.9), during which all patients showed HN improvement without UTI or flank pain development. The median DRF from the final postoperative MAG-3 diuretic renogram was $38.0 \%$ (IQR: $13.3-48.2 \%$ ). Patients did not require surgical interventions other than the ureteral stent removal in LP or RALP cases.

Hypertension was observed in 2 patients. In one case, $\mathrm{OP}$ was performed when the patient (male) was 4.3 years old due to decreased DRF to $25.5 \%$ and UTI development. After the operation, HN improved, and postoperative DRF was $23.3 \%$. Hypertension with systolic blood pressure between 130 and $140 \mathrm{mmHg}$ was observed 7.7 years after surgery. Cardiac echography and electrocardiography revealed no abnormalities, and lifestyle modifications were implemented. The systolic blood pressure decreased to between 120 and $130 \mathrm{mmHg}$ and was maintained without medication. In the other case, UPJO in the HSK was detected after a traumatic renal pelvis rupture at 17 years old. The (male) patient's systolic blood pressure was between 140 and $160 \mathrm{mmHg}$ with a DRF of $4.0 \%$ by MAG-3 renogram. A ureteral stent was inserted, and the systolic blood pressure decreased to approximately $130 \mathrm{mmHg}$ with medication. Two months after the ureteral stent insertion, the DRF increased to $31.9 \%$, and RALP was performed. After RALP, the systolic blood pressure decreased to approximately $110 \mathrm{mmHg}$ without medication. The postoperative DRF was $29.8 \%$. Proteinuria was not observed before or after the operations in all patients.

\section{Discussion}

UPJO is frequently associated with HSK, occurring in $15 \%$ to $33 \%$ of HSK patients (4). The high incidence rate is due to the abnormal location of the ureter over the isthmus, the crossing of multiple aberrant renal vessels over the ureter, HUI in the renal pelvis, and congenital stricture of the UPJ (6). Several UPJ features were reported to cause HSK obstructions. Thus, several surgical techniques have been suggested, including isthmotomy with kidney separation, vascular hitch, endopyelotomy, ureterocalicostomy, and conventional dismembered pyeloplasty (7). Isthmotomy with kidney separation is commonly reported. Yohannes et al. described OP for HSKs by dividing the renal isthmus followed by UPJ reconstruction (4). Kawauchi 
et al. suspected the isthmus to be the UPJO cause and subsequently performed an LP and isthmus transection (8). However, many other studies report positive patient outcomes without isthmus separation, indicating that isthmus separation may not be the best surgical option $(7,9,10)$. Our study results support this hypothesis; during the operations, the isthmus did not appear to be the cause of the obstructions, and all cases had successful results without isthmus separation. Although isthmotomy could be performed successfully with surgical instruments, such as an argon laser, coagulating microwave heat, and electrocauterization, there are risks for bleeding and renal ischemia from cutting the renal isthmus feeding vessels (4). Thus, isthmotomy with kidney separation is not appropriate for HSK patients with UPJOs.

$\mathrm{CV}$ is a known cause of UPJO and was reported in 40 to $80 \%$ of UPJO patients with HSKs (11). Our results are consistent with previous reports, as CVs were observed in $60.0 \%$ of patients. Bleve et al. reported successful results from a laparoscopic vascular hitch operation in three children with HSKs (12). However, the vascular hitch managed the CVs but could not resolve the HUI. In the HSK, the ureter may lie laterally, and a high insertion to the renal pelvis may develop owing to incomplete renal rotation (13). As the UPJ is not located at the dependent position of the renal pelvis, urine cannot drain properly in the lower part of the renal pelvis. Dilatation in the lower renal pelvis also makes an already high UPJ move toward a higher position and may occasionally cause acute complete obstruction of the UPJ. In our study, HUI was observed in $80 \%$ of cases but in only $20 \%$ to $40 \%$ of patients in previous studies $(3,14)$. Our HUI rate may be caused by our definition of HUI, which was defined as when the ureter is not connected with the dependent position of the renal pelvis in retrograde ureteropyelography. A HUI diagnosis may be difficult without retrograde ureteropyelography, and other studies did not define how HUI was diagnosed, making our results difficult to compare with previous studies.

Endopyelotomy was introduced for UPJO management with a success rate of $70 \%$ to $89 \%$ in UPJO patients without HSKs. Nakamura et al. first attempted this procedure in three HSK patients (15), and the success rate reported in a small case series was approximately $66 \%$ to $70 \%$ (16). The presence of CVs in a high percentage of HSKs is responsible for the failure rate (17), and even without $\mathrm{CVs}$, HUIs could not be resolved by endopyelotomy.

Ureterocalicostomy was also performed in HSK patients.
Radford et al. reported four cases of ureterocalicostomy in UPJO patients with HSKs by connecting the mucosa of the lower calyx to the proximal ureter (18). The authors concluded that ureterocalicostomy is a reliable technique for UPJO with anomalous anatomies, such as HSK and recurrent obstructions. When HUIs and CVs occur together, there is minimal space to make a new UPJ in the dependent position of the renal pelvis (between the lower pole parenchyma and the CV) (Figure 1). Subsequently, some studies attempted ureterocalicostomy. However, our results showed that a new UPJ could be created in the small space and that all cases had good outcomes regardless of surgical modality. Ureterocalicostomies have a high risk of lower pole kidney damage and bleeding, and should, therefore, not be considered first. Conventional dismembered pyeloplasty with neo-UPJ formation combined with CVs is a safe and successful method in UPJO patients with HSKs.

Surgical modality is another important issue for UPJO patients with HSKs (19). OP is considered the gold standard modality and has a high success rate $(2,20,21)$. Schuessler et al. performed LP for non-HSKs in 1993, and Janetschek et al. introduced the method for HSKs in $1996(22,23)$. The long anastomotic time and intracorporeal knot tying issues are the main obstacles during the LP procedure in HSK cases owing to the unexpected anatomical vascular findings $(3,8)$. With the introduction of RALP, many of the laparoscopic technique difficulties were solved by providing the surgeon with a three-dimensional view of the surgical field and improved hand dexterity for tissue handling and intracorporeal anastomosis and knotting (24). Chammas et al. introduced RALP as a safe and successful modality for pyeloplasty in HSK patients in 2006 (25). In our study, all cases had successful clinical outcomes regardless of surgical modality. Although a retroperitoneoscopic approach has been used in conventional UPJO cases, this study did not include this approach because of HSK immobilization, the difficult passage to the renal pelvis, and the small surgical field.

As HSKs may frequently develop UPJOs, careful followup is mandatory if the HSK patient has HN. However, there is not yet a suitable follow-up plan for HSK patients without HN. In this study, HN was diagnosed prenatally in only five patients. In the other 15 , there was no HN during the fetal period, or it was missed during the prenatal period. The national health insurance system in Korea was implemented for the entire population in 1989 and covers prenatal ultrasounds during pregnancy. According to a survey in $1994,97.6 \%$ of pregnant women had an 
ultrasound during pregnancy (26). One or two level-II targeted prenatal ultrasounds are covered by the national health insurance system. Therefore, it is assumed that prenatal targeted ultrasounds were performed for most subjects included in this study. In the five prenatal HN cases, it is more likely that ultrasounds were not performed than that the diagnosis was missed. Attention should be given to UPJO in HSK patients, even in cases with no HN, as it may develop later, but a follow-up protocol cannot be clearly defined by this research. Future research should target patients diagnosed with HSKs without HN before birth to determine a suitable follow-up protocol.

This research has the limitation of retrospective research for selection bias. Additionally, our institution is a tertiary referral center, and most of the patients did not attend our hospital during pregnancy but were referred after the UPJO diagnosis. Analyzing the prenatal examination data from the patients included in this study would provide a more accurate analysis of HSKs without HN. Nonetheless, to our knowledge, this study is the largest analysis of HSK patients with UPJOs. Further HSK cohort studies may allow a follow-up protocol to be proposed.

\section{Conclusions}

UPJOs in HSK patients were primarily caused by a HUI and CVs. Dismembered pyeloplasty was successfully performed in all surgical modalities (i.e., OP, LP, and RALP). Special attention must be given to UPJOs in HSK cases, even without HN, as UPJOs can develop over time.

\section{Acknowledgments}

Funding: None.

\section{Footnote}

Reporting Checklist: The authors have completed the STROBE reporting checklist. Available at https://dx.doi. org/10.21037/tau-21-471

Data Sharing Statement: Available at https://dx.doi. org/10.21037/tau-21-471

Conflicts of Interest: All authors have completed the ICMJE uniform disclosure form (available at https://dx.doi. org/10.21037/tau-21-471). The authors have no conflicts of interest to declare.
Ethical Statement: The authors are accountable for all aspects of the work in ensuring that questions related to the accuracy or integrity of any part of the work are appropriately investigated and resolved. This study was conducted in accordance with the Declaration of Helsinki (as revised in 2013). The study was approved by institutional review board of Severance Hospital (4-2020-1078) and individual consent for this retrospective analysis was waived.

Open Access Statement: This is an Open Access article distributed in accordance with the Creative Commons Attribution-NonCommercial-NoDerivs 4.0 International License (CC BY-NC-ND 4.0), which permits the noncommercial replication and distribution of the article with the strict proviso that no changes or edits are made and the original work is properly cited (including links to both the formal publication through the relevant DOI and the license). See: https://creativecommons.org/licenses/by-nc-nd/4.0/.

\section{References}

1. Natsis K, Piagkou M, Skotsimara A, et al. Horseshoe kidney: a review of anatomy and pathology. Surg Radiol Anat 2014;36:517-26.

2. Pitts WR Jr, Muecke EC. Horseshoe kidneys: a 40-year experience. J Urol 1975;113:743-6.

3. Blanc T, Koulouris E, Botto N, et al. Laparoscopic pyeloplasty in children with horseshoe kidney. J Urol 2014;191:1097-103.

4. Yohannes P, Smith AD. The endourological management of complications associated with horseshoe kidney. J Urol 2002;168:5-8.

5. Kang SK, Jang WS, Kim SW, et al. Robot-assisted laparoscopic single-port pyeloplasty using the da Vinci $\mathrm{SP}$ (system: initial experience with a pediatric patient. J Pediatr Urol 2019;15:576-7.

6. Faddegon S, Granberg C, Tan YK, et al. Minimally invasive pyeloplasty in horseshoe kidneys with ureteropelvic junction obstruction: a case series. Int Braz J Urol 2013;39:195-202.

7. Nishi M, Iwamura M, Kurosaka S, et al. Laparoscopic Anderson-Hynes pyeloplasty without symphysiotomy for hydronephrosis with horseshoe kidney. Asian J Endosc Surg 2013;6:192-6.

8. Kawauchi A, Fujito A, Yoneda K, et al. Laparoscopic pyeloplasty and isthmectomy for hydronephrosis of horseshoe kidney: a pediatric case. J Endourol 2005;19:984-6. 
9. Shadpour P, Akhyari HH, Maghsoudi R, et al. Management of ureteropelvic junction obstruction in horseshoe kidneys by an assortment of laparoscopic options. Can Urol Assoc J 2015;9:E775-9.

10. Schuster TK, Jacobs BL, Gayed BA, et al. Preliminary experience with laparoscopic ureteropelvic junction release in the treatment of ureteropelvic junction obstruction. J Endourol 2010;24:393-6.

11. Oderda M, Calleris G, Allasia M, et al. Robot-assisted laparoscopic pyeloplasty in a pediatric patient with horseshoe kidney: surgical technique and review of the literature. Urologia 2017;84:55-60.

12. Bleve C, Bucci V, Conighi ML, et al. Horseshoe kidney and uretero-pelvic-junction obstruction in a pediatric patient. Laparoscopic vascular hitch: A valid alternative to dismembered pyeloplasty? Pediatr Med Chir 2017;39:178.

13. Partin A, Peters C, Kavoussi L, et al. Campbell Walsh Wein Urology, 12th Edition, 731, Elsevier, 2020.

14. Bove P, Ong AM, Rha KH, et al. Laparoscopic management of ureteropelvic junction obstruction in patients with upper urinary tract anomalies. J Urol 2004;171:77-9.

15. Nakamura K, Baba S, Tazaki H. Endopyelotomy in horseshoe kidneys. J Endourol 1994;8:203-6.

16. Jabbour ME, Goldfischer ER, Stravodimos KG, et al. Endopyelotomy for horseshoe and ectopic kidneys. J Urol 1998;160:694-7.

17. Van Cangh PJ, Nesa S, Galeon M, et al. Vessels around the ureteropelvic junction: significance and imaging by

Cite this article as: Elmaadawy MIA, Kim SW, Kang SK, Han SW, Lee YS. A retrospective analysis of ureteropelvic junction obstructions in patients with horseshoe kidney. Transl Androl Urol 2021;10(11):4173-4180. doi: 10.21037/tau-21-471 conventional radiology. J Endourol 1996;10:111-9.

18. Radford AR, Thomas DF, Subramaniam R.

Ureterocalicostomy in children: 12 years experience in a single centre. BJU Int 2011;108:434-8.

19. Lallas CD, Pak RW, Pagnani C, et al. The minimally invasive management of ureteropelvic junction obstruction in horseshoe kidneys. World J Urol 2011;29:91-5.

20. Siqueira TM Jr, Nadu A, Kuo RL, et al. Laparoscopic treatment for ureteropelvic junction obstruction. Urology 2002;60:973-8.

21. Das S, Amar AD. Ureteropelvic junction obstruction with associated renal anomalies. J Urol 1984;131:872-4.

22. Schuessler WW, Grune MT, Tecuanhuey LV, et al. Laparoscopic dismembered pyeloplasty. J Urol 1993;150:1795-9.

23. Janetschek G, Peschel R, Bartsch G. Laparoscopic and retroperitoneoscopic kidney pyeloplasty. Urologe A 1996;35:202-7.

24. Boysen WR, Gundeti MS. Robot-assisted laparoscopic pyeloplasty in the pediatric population: a review of technique, outcomes, complications, and special considerations in infants. Pediatr Surg Int 2017;33:925-35.

25. Chammas M Jr, Feuillu B, Coissard A, et al. Laparoscopic robotic-assisted management of pelvi-ureteric junction obstruction in patients with horseshoe kidneys: technique and 1-year follow-up. BJU Int 2006;97:579-83.

26. Yoo SH, Kang BS, LEE JA, et al. Preventive Medicine, 3rd edition, 924-932, Gyechuk-moonhwasa, 2007. 